\title{
Análisis de NIIF 9 - Instrumentos Financieros \\ desde una perspectiva industrial
}

\section{Carlos Gabriel Parrales Choez, Franklin Duberlí Castillo Llanos}

Facultad de Ciencias Administrativas

Universidad de Guayaquil

El artículo muestra el papel y la dinámica de la Norma Internacional de Información Financiera 9 denominada «Instrumentos Financieros». Esta norma sustituye a la NIC 39 - Instrumentos Financieros: Reconocimiento y Medición, por lo cual es importante conocer esta normativa dentro de las diferentes entidades existentes en un país, en este caso, las pymes del sector industrial. De esta manera, es posible ayudar en la correcta contabilización y diferenciación de los instrumentos financieros.

El objetivo es establecer los principios para la información financiera sobre activos y pasivos financieros, de forma que se presente información útil y relevante a los usuarios de los estados financieros para la evaluación de los importes, el calendario y la incertidumbre de los flujos de efectivo futuros de la entidad. Esto les facilitará la comprensión de la información financiera a las pequeñas y medianas empresas, al revelar los detalles correspondientes de esta norma para su respectiva asimilación y la creación de un modelo de aplicación que las involucre. Cabe anotar que estos parámetros de contabilización deben ser conocidos a la perfección por los responsables de la entidad, en este caso, la persona encargada del registro de las transacciones correspondientes durante un determinado período. Dicha persona asumiría el rol del contador público autorizado, quien debe tener los conocimientos y la experiencia suficientes para aplicar correctamente los procedimientos, y brindar seguridad en el proceso de registro y uso de la norma.

Palabras clave: procedimientos, activos financieros, principios, medición, valoración, valor

\section{Analysis of IFRS 9 Financial Instruments from an industrial perspective}

The article shows the role and dynamics of International Financial Reporting Standard No. 9 called Financial Instruments, this standard replaces IAS 39 "Financial Instruments; Recognition and measurement", which is why the knowledge of these regulations within the different entities in a country, in this case SMEs in the industrial sector, is important to help in the correct accounting and differentiation of financial instruments, with the objective of establishing the principles for financial reporting on financial assets and financial liabilities, so that useful and relevant information is presented to the users of the financial statements for the assessment of the amounts, timing and uncertainty of the entity's future cash flows. This will facilitate the understanding of financial information by small and medium-sized companies, explaining this rule with the corresponding details for their respective assimilation and creating an application model that involves them. These accounting parameters must be perfectly known by the person in charge of the entity in this case the person responsible for recording the corresponding transactions during a certain period who is defined as an Authorized Public Accountant, who must have sufficient knowledge and experience to correctly apply procedures and provide security in the registration process.

Keywords: Procedures, financial assets, principles, measurement, valuation, value 


\section{Análise da IFRS 9 Instrumentos Financeiros do ponto de vista industrial}

$\mathrm{O}$ artigo mostra o papel e a dinâmica da Norma Internacional de Relato Financeiro $\mathrm{N}^{\circ} 9$ denominada Instrumentos Financeiros, esta norma substitui a IAS 39 «Instrumentos Financeiros; reconhecimento e mensuração» é por isso que o conhecimento desses regulamentos é importante dentro das diferentes entidades de um país, neste caso as PME do setor industrial, para ajudar na correta contabilização e diferenciação de instrumentos financeiros, com o objetivo de estabelecer os princípios para relatórios financeiros sobre ativos e passivos financeiros, de forma que informaçóes úteis e relevantes sejam apresentadas aos usuários das demonstraçóes financeiras para a avaliação dos valores, tempestividade e incerteza dos fluxos de caixa futuros da entidade. Isso facilitará a compreensão da informação financeira para pequenas e médias empresas, revelando os detalhes correspondentes desta norma para sua respectiva assimilaçáo, criando um modelo de aplicação que os envolva. Estes parâmetros contábeis devem ser perfeitamente conhecidos pelos gestores da entidade, neste caso a pessoa encarregada de registrar as transaçôes correspondentes durante um determinado período a quem é definido como um Contador Público Autorizado, que deve ter conhecimento e experiência suficientes. aplicar corretamente os procedimentos e fornecer segurança no processo de registro e uso da norma.

Palavras-chave: procedimentos, ativos financeiros, princípios, mensuração, valorização, valor

\section{Introducción}

Frente a los procesos de globalización, «las empresas necesitan crecer y encontrar oportunidades de negocio trascendiendo fronteras nacionales» (Higuera, 2014). Además, el sistema financiero se ha integrado a tal nivel que cualquier suceso económico, social y político tiene repercusiones en los sectores económicos a nivel mundial. La Junta de Estándares Internacionales de Contabilidad (IASB, por sus siglas en inglés), ente privado compuesto por profesionales de la industria financiera y contadores, publicó los nuevos estándares de contabilidad y de revelación de información como una alternativa a los problemas y las limitaciones que genera la heterogeneidad de los sistemas existentes alrededor del mundo. En la fecha del 24 de julio de 2014, emitió la versión definitiva de la NIIF 9 - Instrumentos Financieros, normativa que tiene como fecha de aplicación obligatoria en las entidades a partir del 1 de enero de 2018.
Esta norma se elaboró en tres fases: la primera estuvo dirigida a la clasificación y medición; la segunda, a la medición de deterioro; y la tercera, orientada a la contabilidad de coberturas. El objetivo consistía en mejorar la presentación de la información financiera sobre instrumentos financieros, abordando preocupaciones que surgieron durante crisis financieras. En particular, este ha sido un modelo más prospectivo para el reconocimiento de las pérdidas esperadas en los activos financieros.

En relación con ello, la nueva normativa establece criterios que se basan en principios y no en reglas, como lo planteaba la NIC 39, que permitía la clasificación de activos financieros de acuerdo con el modelo de negocio y según la naturaleza de los flujos de efectivo del instrumento. Por su parte, en los pasivos financieros, se distingue claramente en el tratamiento contable las variaciones del valor razonable provenientes del riesgo de mercado y de crédito, a la vez que es posible reclasificarlas de acuerdo con los cambios en el modelo de negocio. 
Para Jhonathan Higuera, «quizá el cambio más radical con las nuevas normas viene dado por su reorientación hacia la utilidad de la información para los inversionistas» (2014, p. 2). En la práctica, la modificación se refleja en la introducción del concepto «valor razonable», requerido para valorar instrumentos derivados, valores de deuda, y otros activos o pasivos.

Según Martha Arias Bello (2014), en lo que respecta a la clasificación y la medición de los activos financieros, «si un instrumento no tiene flujos contractuales definidos, como por ejemplo capital e intereses, siempre se clasificarán y medirán a valor razonable con cambios en el resultado». No obstante, se permite que, para los instrumentos de patrimonio, las variaciones afecten el otro resultado integral, decisión que será irrevocable.

Si la entidad espera recoger flujos contractuales del activo financiero al vencimiento, estos deberán ser clasificados al costo amortizado. En cambio, si el activo financiero se mantiene dentro de un modelo de negocio cuyo objetivo es obtener los flujos de efectivo contractuales y vender simultáneamente los activos financieros, y adicionalmente los términos contractuales del activo financiero dan lugar en fechas determinadas a los flujos de efectivo por concepto de principal e intereses, estos instrumentos se clasificarán y medirán a valor razonable con efectos en el otro resultado integral. Entonces, para la clasificación y la medición de estos instrumentos a valor razonable con efectos en resultados, la decisión depende de que se proporcione información más fiable y relevante, como, por ejemplo, en el caso de reducir o eliminar asimetrías contables, cuando el instrumento hace parte de una relación de cobertura.

Según Chorafas (2007), otro de los cambios importantes que las NIIF trajeron a la contabilidad corporativa refiere a los activos y pasivos financieros requeridos en el balance general a valor de mercado»; la medición del Good Will (también, denominado plusvalía, crédito mercantil o fondo de comercio) que utiliza el valor recuperable estimando los retornos sobre la inversión; la cuantificación de activos intangibles como patentes, marcas registradas o proyectos de investigación y desarrollo; la contabilización en el estado anual de pérdidas y ganancias de activos y pasivos pensionales; y la revelación plena de las compensaciones a ejecutivos.

Por su naturaleza, la NIIF 9 tiene que aplicarse de forma retroactiva, con ciertas excepciones, aunque no se exija reexpresar los períodos comparativos. En ese sentido, se puede afirmar que el enfoque es totalmente adverso al de NIC 39, pero, en conjunto, sin lugar a dudas, existirán mejoras en el modelo actual.

\section{Importancia del problema}

El desconocimiento de las pymes industriales acerca de la contabilización y de la diferenciación de los instrumentos financieros constituye un problema dentro de los alcances contables, por lo cual es importante que dichas empresas conozcan la normativa vigente para su correcta aplicación. De esta manera, es posible ayudar a la adecuada contabilización y al reconocimiento de los instrumentos financieros.

Frente a ello, esta investigación abarca el análisis de NIIF 9 - Instrumentos Financieros para la creación de un modelo de aplicación dentro de las empresas industriales. En ese marco, primero, es necesario establecer los principios de información financiera sobre activos financieros y pasivos financieros. En segundo lugar, se debe identificar los lineamientos para crear un modelo de aplicación y facilitar la compresión de la información financiera a los usuarios. Por último, 
es preciso definir el reconcomiendo inicial y posterior de los activos financieros en el reemplazo de la NIC 39 para las empresas industriales a través de la aplicación de la NIIF 9.

\section{Metodología}

\subsection{Delimitación de la investigación}

La presente investigación se realizó en el sector industrial de la ciudad de Guayaquil durante el período de 2016 debido a la necesidad de obtener capital, liquidez operativa y rentabilidad en este tipo de organizaciones. En función de esta, se revisará documentalmente las normas emitidas por organismos involucrados en el proceso de regulación y control de los activos y pasivos financieros, utilizando como fuente de recolección de datos diversos documentos generales y estadísticos publicados en sitios web de instituciones públicas y privadas.

\subsection{Métodos de investigación}

En el transcurso de la investigación, se espera entender a profundidad la problemática que enfrentan las pequeñas, medianas y grandes empresas del sector industrial en cuanto al desconocimiento de la NIIF 9. Para proceder a resolver la situación, mediante el análisis y el estudio estratégico de dicha normativa, se utilizará métodos investigativos que permitan lograr el objetivo pretendido. El método hipotético-deductivo o de contrastación de las hipótesis se basa en demostrar la verdad o falsedad de las hipótesis, puesto que estas no pueden ser demostradas o comprobadas directamente. La esencia de este método investigado consiste en verificar si la verdad o la falsedad del enunciado básico muestra la verdad o la falsedad de las hipótesis que ponemos a prueba (Cazau, 2012). A partir de ello, esta propuesta metodológica tiene como finalidad poner a prueba el tema de investigación de la manera más exigente posible aplicada al sector industrial.
Otra de las técnicas empleadas es la revisión documental. Según Arias, «[...] la investigación documental es un proceso basado en la búsqueda, recuperación, análisis, crítica e interpretación de datos secundarios, es decir, los obtenidos y registrados por otros investigadores en fuentes documentales: impresas, audiovisuales o electrónicas» (2012, p. 27). Como en toda investigación, el propósito de este diseño es el aporte de nuevos conocimientos; sin embargo, es importante aclarar que, aun cuando las fuentes documentales aportan datos secundarios, estas a su vez se clasifican en fuentes documentales primarias (obras originales) y fuentes documentales secundarias (trabajos en los que se hace referencia a la obra de un autor).

\subsection{Población}

Tamayo y Tamayo definen la población como un «[...] conjunto finito o infinito de personas, casos o elementos, que presentan características comunes» (2012, p. 180). El término población en nuestro estudio se refiere específicamente al número de empresas que constan en el directorio de la provincia del Guayas de todos los sectores productivos en el año 2016, información que fue tomada de la Superintendencia de Compañías, Valores y Seguros (2017), como se muestra en el gráfico 1 .

Tabla 1. Número de empresas por su tamaño, Guayas

\begin{tabular}{|l|c|c|}
\hline \multicolumn{2}{|c|}{ Indicadores Guayas - Sector industrial } \\
\hline \multicolumn{2}{|c|}{ Número de empresas por su tamaño } \\
\hline Tamaño de empresa & Año 2016 & $\%$ \\
\hline Microempresa & 620 & 30 \\
Pequeña empresa & 638 & 31 \\
Mediana empresa & 819 & 39 \\
\hline Total & 2077 & 100 \\
\hline
\end{tabular}

Fuente: Superintendencia de Compañías, Valores y Seguros (2017). 
Gráfico 1. Representación porcentual de las empresas según su tamańo (Guayas)

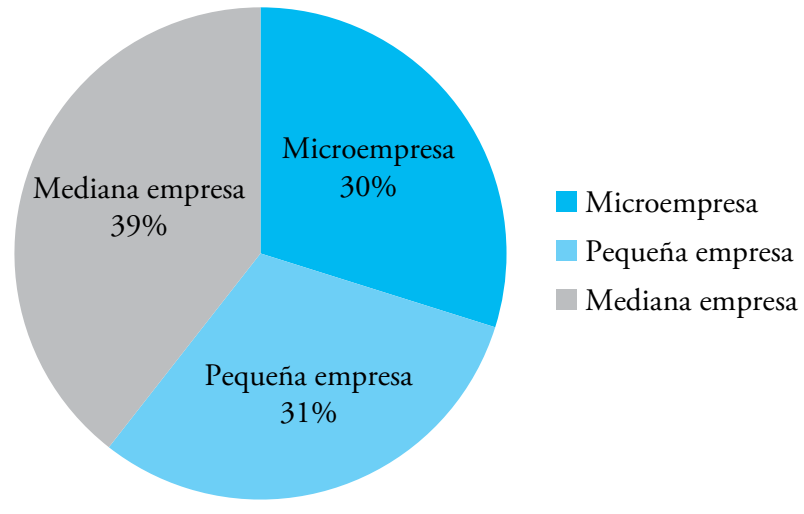

Fuente: Superintendencia de Compañías, Valores y Seguros (2017).

\subsection{Diseño muestral}

Arias indica que la muestra «[...] es un subconjunto representativo y finito que se extrae de la población accesible» (2012, p. 83). El objetivo de seleccionar una muestra responde a que, pocas veces, se puede medir a la población en su totalidad. Por este motivo, una vez definida la unidad de análisis, se procede a delimitar el objeto de estudio. En este caso, la muestra abarcará el número de empresas que constan en el directorio de la provincia del Guayas, específicamente, el sector manufacturero o industrial del año 2016, información que fue tomada de la Superintendencia de Compañías, Valores y Seguros (2017), como se muestra en el gráfico 2.

Tabla 2. Número de empresas por su tamańo (Guayaquil)

\begin{tabular}{|l|c|c|}
\hline \multicolumn{2}{|c|}{ Indicadores Guayaquil - Sector industrial } \\
\hline Número de empresas por su tamaño \\
\hline Tamaño de empresa & Año 2016 & $\%$ \\
\hline Microempresa & 572 & 40 \\
Pequeña empresa & 586 & 41 \\
Mediana empresa & 273 & 19 \\
\hline Total & 1431 & 100 \\
\hline
\end{tabular}

Fuente: Superintendencia de Compañías, Valores y Seguros (2017).
Gráfico 2. Representación porcentual de las empresas según su tamaño (Guayaquil)

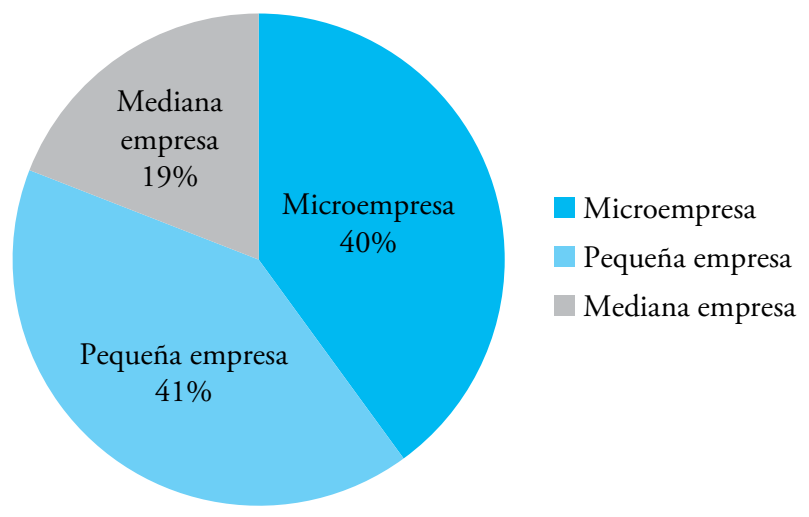

Fuente: Superintendencia de Compañías, Valores y Seguros (2017).

\subsection{Análisis}

Como resultado de este análisis basado en la información recopilada a través de gráficos estadísticos, se puede evidenciar claramente que las pymes del sector industrial representan gran parte de la economía tanto en la provincia del Guayas como en su cantón Guayaquil. En este contexto, la aplicación de la NIIF 9 resulta trascendental para el correcto desenvolvimiento de este tipo de entidades no solo en el curso normal del negocio, sino también para aprender de la utilización de estos instrumentos. De este modo, se mejorará el proceso de registro, reconocimiento, medición, valorización, deterioro, tanto de los activos como de los pasivos financieros que se producen en los diferentes sucesos contables durante un período determinado. En relación con ello, el propósito principal de este artículo es mostrar los diferentes aspectos representativos dentro de esta norma, que permitan una mejor comprensión y asimilación de los temas tratados en la misma, así como una mejor aceptación de los lineamientos emitidos por las instituciones controladoras de los procesos contables tanto nacionales como extranjeros, en función 
de lo cual se procede a explicar de forma explícita las NIIF 9.

\section{Resultados y discusión}

\subsection{Generalidades}

\subsubsection{Normas Internacionales de Información Financiera (NIIF)}

Las Normas Internacionales de Información Financiera (NIIF), según el International Accounting Standards Board (IASB), «[...] son un conjunto de normas contables de carácter mundial de alta calidad, comprensibles y de obligatorio cumplimiento, que exijan información transparente y de alta calidad en la información financiera» (2017, p. 5). Su objetivo es ayudar a los participantes en los mercados de capitales de todo el mundo y a otros usuarios a tomar decisiones económicas. Bajo las NIIF, las empresas ya no pueden limitarse únicamente a mostrarle al usuario de sus estados financieros datos de carácter cuantitativo sin introducirlos en un contexto que evidencie el porqué de dichas cifras. La información sobre las políticas, los objetivos y los procedimientos de administración y manejo del capital deben ser reveladas, incluido lo que para la firma se define como capital (Chorafas, 2007).

\subsubsection{NIIF 9 - Instrumentos financieros}

Según el IASB, el objetivo de esta norma es «[...] establecer los principios para la información financiera sobre activos financieros y pasivos financieros, de forma que se presente información útil y relevante para los usuarios de los estados financieros para la evaluación de los importes, calendario e incertidumbre de los flujos de efectivo futuros de la entidad» (2014, p. 2). Así, las entidades del sector industrial podrán presentar la información acerca de los instrumentos financieros de una forma más comprensiva y entendible, lo que facilita la aplicación de esta normativa en el ámbito contable.

Esta NIIF tiene un alcance muy similar a la NIC 39, pero, en relación con el deterioro, sí es más amplio a su predecesora e incluye activos, como cuentas por cobrar por arrendamientos y los activos contractuales de NIIF 15. De igual forma, aún sigue existiendo la llamada excepción por uso propio, por lo que ciertos contratos para la compra o la venta de partidas no financieras quedan fuera del alcance normativo evaluado. Sin embargo, este es un aspecto práctico que muchas empresas manifestaron en su momento. Por ejemplo, empresas que transforman la materia prima pueden cubrir la exposición al riesgo económico de sus contratos de compra-venta de existencias con derivados de materias primas que se midan a valor razonable con cambios en pérdidas y ganancias. Además, para muchas empresas, la contabilidad de cobertura podría ser una opción para evitar asimetrías contables. En ese sentido, incluir esta opción de valoración a valor razonable en contratos de uso propio representa una solución para lograr mayor coherencia entre el reflejo contable y la gestión económica del riesgo.

En el presente artículo, vamos a desarrollar todas las novedades de la norma; explicaremos los criterios de clasificación y valoración de los activos financieros, así como las novedades aplicables a los contratos de compra-venta de partidas no financieras del sector industrial. Cabe anotar que la norma elimina ciertas categorías, como son préstamos y partidas a cobrar, inversiones a vencimiento y disponibles para la venta: solo deja como instrumentos financieros valorados a coste amortizado; a valor razonable con cambios en otro resultado global; y valor razonable con cambios en resultados, ya sean designados o mantenidos para negociar, como se muestra a continuación. 


\section{Tabla 3. NIIF 9 - Instrumentos financieros: criterios de valoración}

\begin{tabular}{|c|c|c|}
\hline \multicolumn{3}{|c|}{ Criterios de clasificación y valoración de los activos financieros } \\
\hline Instrumento financiero & Valoración inicial & Valoración posterior \\
\hline $\begin{array}{l}\text { Activos financieros mantenidos para } \\
\text { cobrar los flujos contractuales y las } \\
\text { cláusulas contractuales dan lugar, } \\
\text { en fechas determinadas, a flujos que } \\
\text { están solamente basados en el prin- } \\
\text { cipal e intereses sobre el principal } \\
\text { pendiente, y se mantienen en un } \\
\text { modelo de negocio cuyo objetivo } \\
\text { es mantener los activos financieros } \\
\text { para cobrar los flujos contractuales. }\end{array}$ & $\begin{array}{l}\text { Valor razonable, inclui- } \\
\text { dos costes de transacción. }\end{array}$ & $\begin{array}{l}\text { - Coste amortizado. } \\
\text { - Devengo de intereses en resultados por el método del tipo de interés } \\
\text { efectivo. } \\
\text { - Las diferencias de cambio se reconocen en resultados. } \\
\text { - Las pérdidas y reversiones del deterioro se reconocen en resultados. }\end{array}$ \\
\hline $\begin{array}{l}\text { Activos financieros mantenidos para } \\
\text { cobrar los flujos contractuales y las } \\
\text { cláusulas contractuales dan lugar, } \\
\text { en fechas determinadas, a flujos que } \\
\text { están solamente basados en el prin- } \\
\text { cipal e intereses sobre el principal } \\
\text { pendiente, y se mantienen en un } \\
\text { modelo de negocio cuyo objetivo } \\
\text { se alcanza mediante el cobro de los } \\
\text { flujos contractuales y la venta de los } \\
\text { activos financieros. }\end{array}$ & $\begin{array}{l}\text { Valor razonable, inclui- } \\
\text { dos costes de transacción. }\end{array}$ & $\begin{array}{l}\text { - Valor razonable, con cambios en otro resultado global. } \\
\text { - Devengo de intereses en resultados por el método del tipo de interés } \\
\text { efectivo. } \\
\text { - Las diferencias de cambio se reconocen en resultados. } \\
\text { - Las pérdidas y las reversiones del deterioro se reconocen en resulta- } \\
\text { dos, pero con abono o cargo al otro resultado global, y no reducen } \\
\text { o aumentan el valor del activo financiero en el estado de situación } \\
\text { financiera. } \\
\text { - Cuando el activo financiero se da de baja, los importes diferidos en } \\
\text { otro resultado global se reclasifican a resultados. }\end{array}$ \\
\hline Instrumentos de patrimonio & $\begin{array}{l}\text { Valor razonable (inclu- } \\
\text { yendo costes de tran- } \\
\text { sacción, si se opta por el } \\
\text { registro de los cambios de } \\
\text { valoración en otro resul- } \\
\text { tado global) }\end{array}$ & $\begin{array}{l}\text { Valor razonable con cambios en resultados, excepto que se pueda optar } \\
\text { por reconocer las variaciones en otro resultado global: La opción es } \\
\text { aplicable instrumento a instrumento. Si el instrumento se reconoce a } \\
\text { valor razonable con cambios en otro resultado global, los dividendos } \\
\text { se reconocen como ingresos, salvo que representen un reembolso de } \\
\text { la inversión. En este último caso, los dividendos se reconocen en otro } \\
\text { resultado global. Los importes registrados en otro resultado global no } \\
\text { se reclasifican a resultados ni por enajenación, ni por deterioro, sin } \\
\text { perjuicio de reclasificarlos a reservas. }\end{array}$ \\
\hline $\begin{array}{l}\text { Resto de activos financieros, inclui- } \\
\text { dos los designados a valor razonable } \\
\text { con cambios en resultados }\end{array}$ & $\begin{array}{l}\text { Valor razonable. Los } \\
\text { costes de transacción se } \\
\text { reconocen en resultados a } \\
\text { medida que se devengan. }\end{array}$ & $\begin{array}{l}\text { Valor razonable con cambios en resultados: No se requiere presentar } \\
\text { separadamente los intereses y los dividendos de estos instrumentos. }\end{array}$ \\
\hline $\begin{array}{l}\text { Pasivos financieros a coste amorti- } \\
\text { zado }\end{array}$ & $\begin{array}{l}\text { Valor razonable, menos } \\
\text { costes de transacción. }\end{array}$ & $\begin{array}{l}\text { - Coste amortizado. } \\
\text { - Devengo de intereses por el método del tipo de interés efectivo. } \\
\text { - Las diferencias de cambio se reconocen en resultados. }\end{array}$ \\
\hline $\begin{array}{l}\text { Pasivos financieros a valor razonable } \\
\text { con cambios en resultados: Nego- } \\
\text { ciación }\end{array}$ & $\begin{array}{l}\text { Valor razonable. Los } \\
\text { costes de transacción se } \\
\text { reconocen en resultados a } \\
\text { medida que se devengan. }\end{array}$ & $\begin{array}{l}\text { Valor razonable con cambios en resultados: No se requiere presentar } \\
\text { separadamente los intereses de estos instrumentos. }\end{array}$ \\
\hline $\begin{array}{l}\text { Pasivos financieros a valor razonable } \\
\text { con cambios en resultados: Desig- } \\
\text { nados }\end{array}$ & $\begin{array}{l}\text { Valor razonable. Los } \\
\text { costes de transacción se } \\
\text { reconocen en resultados a } \\
\text { medida que se devengan. }\end{array}$ & $\begin{array}{l}\text { Valor razonable, que registra la variación del valor por el riesgo de cré- } \\
\text { dito en otro resultado global, salvo que suponga crear o aumentar una } \\
\text { asimetría contable, y el resto en resultados. El importe diferido en otro } \\
\text { resultado global no se reclasifica posteriormente a resultados. No se } \\
\text { requiere presentar separadamente los intereses de estos instrumentos. }\end{array}$ \\
\hline
\end{tabular}




\subsubsection{Criterios de valoración aplicables a los activos financieros en cuanto a los contratos en las pymes del sector industrial}

Esta norma ha modificado ciertos criterios de contabilización; a partir de ello, en el caso de las pymes del sector industrial, en los contratos de compra y venta de una partida no financiera, se puede liquidar por el neto en efectivo o con otro instrumento financiero, o mediante el intercambio de instrumentos financieros. Como muestra de que los contratos son instrumentos, los mismos se encuentran bajo el alcance normativo, salvo que los contratos se hayan firmado, y se continúe manteniendo con el propósito de recibir o entregar una partida no financiera de acuerdo con la expectativa de compra, venta o uso de la entidad. No obstante, la norma permite que una entidad designe contratos como valorados a valor razonable con cambios en resultados. Esto se puede realizar al inicio del contrato, y solo si elimina o reduce una inconsistencia de reconocimiento que surgiría en caso de que el contrato no se designara como tal, precautelando la aplicación integral de NIIF 9. A continuación, se muestra las diversas novedades en cuanto al proceso de los contratos.

\section{Gráfico 3. Tratamiento contable de los contratos de compra-venta de partidas no financieras}

¿Se puede liquidar el contrato por diferencias en efectivo, incluida liquidación neta con otro instrumento financiero, o mediante el intercambio de instrumentos financieros o mediante la entrega de materias primas que son fácilmente convertibles en efectivo?

\section{Sí}

¿Se firmó el contrato y se sigue manteniendo para el uso, compra o venta esperada por la entidad?

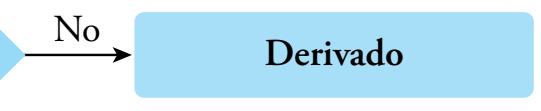

\section{Sí}

¿Eliminaría reruciría significativamente la contabilización a valor razonable una asimetría contable?

\section{Sí}

¿Se ha elegido la opción del valor razonable?

No

Valor razonable con cambios en resultados 


\subsubsection{El modelo de negocio en una pyme industrial}

Para Matiz y Asociados, el modelo de negocio «[...] es una representación simplificada de la lógica del negocio, es decir, es la descripción de la forma como cada negocio ofrece sus productos o servicios a los clientes, como llega a estos, su relación con ellos y cómo la empresa gana dinero» (2015). Por razones coherentes, en una pyme industrial, se establece el modelo de negocio a través de la evaluación realizada por el personal clave de la entidad, es decir, la administración. Este modelo busca reflejar y gestionar los grupos de activos financieros de forma conjunta para alcanzar un objetivo independiente que difiere de la unidad administrativa. Puesto que existen diferentes carteras, una de las más comunes en el sector industrial es la de cuentas por cobrar, que, al aplicar NIIF 9, se convierte en instrumentos financieros a costo amortizado.

\subsection{Aplicación NIIF 9 - Instrumentos Financieros}

\subsubsection{Modelo de aplicación de la NIIF 9 en las pymes industriales}

Las diversas pymes industriales hacen referencia al modelo de negocio que toma en cuenta la forma de gestionar de los activos financieros para generar flujos de efectivo que serán el resultado del cobro flujos contractuales, la venta de activos financieros o ambos. Como consecuencia, la evaluación se realiza sobre la base de escenarios positivos que tengan razonabilidad de suceso, obviando cualquier evento de tipo pesimista. Un ejemplo claro se da cuando la entidad supone la venta de una cartera de activos financieros en situaciones extremas; no por este escenario el modelo de negocio va a ser afectado. La entidad debe aplicar el juicio profesional al evaluar el modelo de negocio para gestionar activos financieros, y dicha evaluación no

Gráfico 4. Aspectos del modelo de negocio

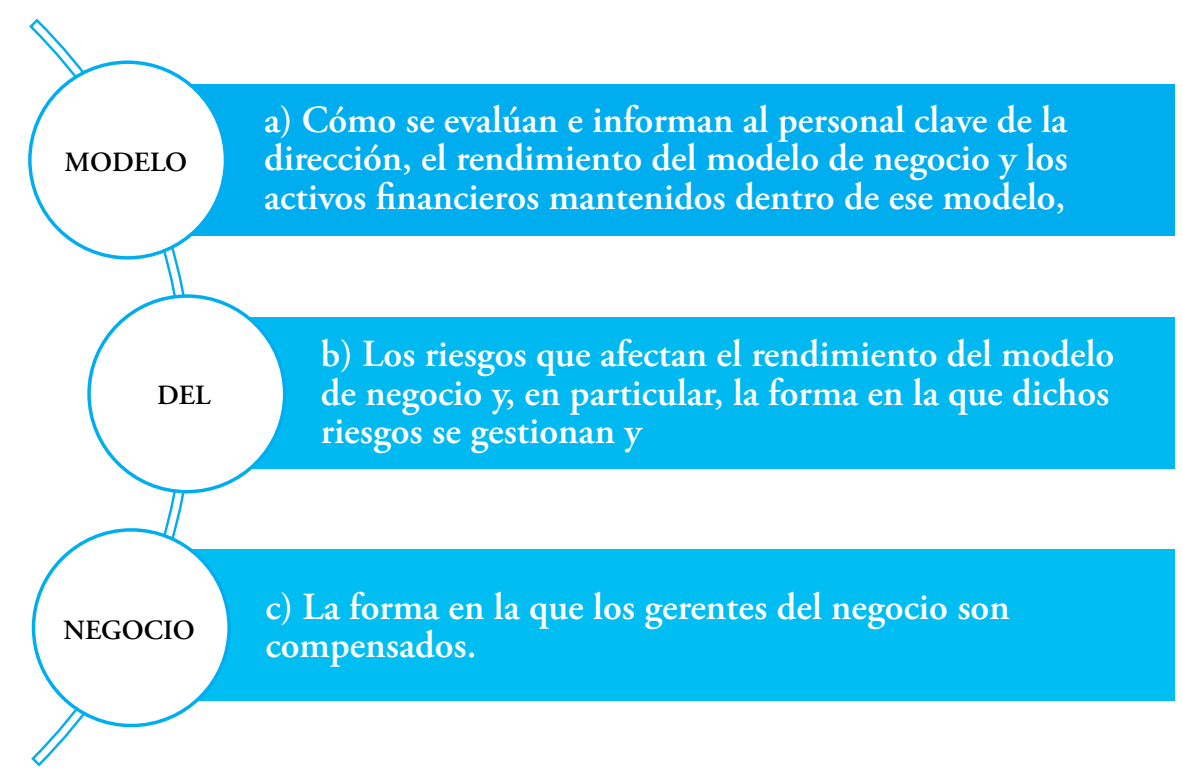


se realiza por un factor individual o actividad. En ese proceso, se debe considerar toda la evidencia relevante que está disponible en la fecha de evaluación, la cual incluye los siguientes elementos, aunque no está limitada a estos.

\subsection{Reconocimiento y medición}

\subsubsection{Reconocimiento y baja de cuentas}

Según Deloitte (2016), los criterios de reconocimiento «[...] son similares a los existentes hasta la fecha en NIC 39. Es decir, una entidad reconoce un activo o pasivo financiero cuando se convierte en parte de las cláusulas contractuales de un instrumento". Sin embargo, los requisitos para baja en cuentas de la NIC 39 se han trasladado sin alteraciones prácticamente a la NIIF 9, incluido el árbol de decisión sobre las bajas de activos. Tomando en cuenta lo antes mencionado, para Angélica Ferrer de la Hoz (2013), en la práctica, "las reglas para dar de baja a un pasivo financiero se mantienen al igual que los criterios contables relacionados con el intercambio de deuda entre un mismo prestamista y prestatario, aplicando el análisis correspondiente denominado 'test del 10' de NIC 39». Sin embargo, es necesario mencionar que las cuentas por cobrar nacen de una venta a crédito. Es decir, por un lado, se genera un derecho de crédito para el cliente; $y$, por otro, una obligación de deuda para el vendedor. Por este motivo, la empresa contabilizará un activo o pasivo financiero solo cuando se convierta en una parte del contrato, lo que da origen a un instrumento financiero junto a su aplicación a través de NIIF 9.

En cuanto a la medición inicial del instrumento de deuda corriente, este proceso se realizará sobre la base del precio de la transacción, incluidos los costos de transacción. En las transacciones de financiación, se utilizará al valor presente. En el primer caso, su medición posterior se realizará de acuerdo con el importe no descontado del efectivo, mientras que, en el segundo, al valor presente. Cabe considerar que la metodología para la medición por deterioro debe asegurar que no se reconozcan pérdidas por deterioro en el momento del reconocimiento inicial de un activo. Con respecto a ello, a continuación, presentamos dos casos de medición inicial y posterior según NIIF 15, que tienen relación con el nacimiento del instrumento financiero.

\subsubsection{Primer caso: Medición inicial y posterior de instrumentos de deuda (clientes)}

El 1 octubre de 2014, la empresa XYZ ha vendido a crédito por importe de US\$1500. El vencimiento de dicho crédito comercial es inferior al primer año.

Tabla 4. Medición inicial del 1 de octubre de 2014 - Precio de la transacción

\begin{tabular}{|c|c|c|c|}
\hline Fecha & Detalle & Debe & Haber \\
\hline $01 / 10 / 2014$ & Clientes & 1500,00 & \\
\hline & Venta de mercaderías & & 1500,00 \\
\hline
\end{tabular}

La medición posterior (31 de diciembre de 2014) es al importe no descontado del efectivo que se espera recibir, que corresponde a US\$1500 siempre y cuando no haya deterioro. Contablemente, no es necesario hacer nada.

\subsubsection{Segundo caso: Medición inicial y posterior de instrumentos de deuda (clientes a largo plazo)}

Tomando los datos del supuesto 1, se estima que el cobro se producirá el 1 de enero de 2017. El tipo de interés de mercado corresponde al 5\%.

- $\mathrm{VP}=1.500 /(1+0,05)^{\wedge} 27 / 12=\mathrm{US} \$ 1344,05$ 
Tabla 5. Medición inicial de octubre de 2016 A valor presente de los pagos futuros descontados

\begin{tabular}{|c|c|c|c|}
\hline Fecha & Detalle & Debe & Haber \\
\hline $01 / 10 / 2014$ & Clientes a largo plazo & 1344,05 & \\
\hline & Venta de mercaderías & & 1344,05 \\
\hline
\end{tabular}

La medición posterior (31 de diciembre de 2014) también es a valor presente de los pagos futuros descontados.

- $\mathrm{VP}=1500 /(1+0,05)^{\wedge} 24 / 12=\mathrm{US} \$ 1360,54$

Entre la medición posterior y la inicial, la diferencia equivale a la siguiente operación: 1360,53 - 1344,05 $=$ US\$16,48.

Tabla 6. Registro de la diferencia entre la medición posterior e inicial

\begin{tabular}{|c|c|c|c|}
\hline Fecha & Detalle & Debe & Haber \\
\hline $01 / 10 / 2014$ & Clientes & 16,48 & \\
\hline & Ingresos financieros & & 16,48 \\
\hline
\end{tabular}

\subsection{Clasificación y valoración de activos financieros}

La NIIF dentro de sus parámetros emitidos por el IASB (2014) requiere que los activos financieros se clasifiquen en el momento de su registro inicial en tres categorías: a su coste amortizado, a valor razonable con cambios en otro resultado integral, o a valor razonable con cambios en pérdidas y ganancias. Esto se debe realizar sobre la base de los dos siguientes aspectos:

- Las características de los flujos de efectivo contractuales del activo financiero

- El modelo de negocio de la entidad para gestionar sus activos financieros

\subsubsection{Características de los fujos contractuales}

Si los flujos de efectivo contractuales del activo son solamente pagos de principal e intereses, los objetivos del modelo de negocio serán los siguientes:

- Uno de ellos consiste en mantener el activo para cobrar sus flujos de efectivo contractuales utilizando la categoría de valoración del coste amortizado; en caso de asimetría contable, se utilizará la opción voluntaria de valor razonable con cambios en pérdidas y ganancias.

- El propósito es mantener el activo para cobrar tanto sus flujos de efectivo contractuales como venderlo, utilizando la categoría de valoración del valor razonable con cambios en otro resultado integral y su reclasificación posterior a pérdidas y ganancias. En caso de asimetría contable, se utilizará la opción voluntaria de valor razonable con cambios en pérdidas y ganancias.

- La finalidad es utilizar otras estrategias, como el trading, a través de valor razonable con cambios en pérdidas y ganancias.

Si los flujos de efectivo contractuales del activo no son solamente pagos de principal e intereses, se utilizará la categoría de valoración del valor razonable con cambios en pérdidas y ganancias. Además, solo para instrumentos de patrimonio, se tiene la posibilidad voluntaria de utilizar la categoría del valor razonable con cambios en otro resultado integral sin reclasificación posterior a pérdidas y ganancias.

En la práctica, evaluar la naturaleza de los flujos de efectivo contractuales permite determinar la medida en que los flujos son solamente pagos del principal e intereses. Esto tiende a complicación siempre y cuando el instrumento sea más sofisticado. En todo 
caso, la NIIF 9 tiene más pormenores técnicos a analizar, a los cuales se debe prestar la mayor atención posible para su correcta percepción.

\subsection{Deterioro}

La NIIF 9 lleva consigo un nuevo proceso de deterioro basado en la pérdida esperada, lo que se diferencia de la NIC 39, que refiere a la pérdida incurrida. Este nuevo modelo es aplicable en los siguientes elementos detallados a continuación:

- Activos financieros valorados al coste amortizado

- Activos financieros de deuda a valor razonable con cambios en otro resultado integral

- Cuentas por cobrar por arrendamientos en el alcance de su norma

- Activos contractuales en el alcance de NIIF 15 Ingresos Derivados de Contratos de Clientes

- Ciertos contratos de garantía financiera y compromisos de préstamo, excepto si se valoran a valor razonable con cambios en pérdidas y ganancias

\subsection{Modelo de aplicación por deterioro de valor de los activos financieros}

El 1 de enero de 2016 la empresa XYZ compró una inversión de deuda que midió a la par por US\$ 500 000. A la fecha, existe una probabilidad del $3 \%$ de que el prestatario entre en incumplimiento, lo que generará una pérdida de $100 \%$. Al 31 de diciembre de 2016, se espera que el prestatario no cumpla con las condiciones del préstamo y existe una probabilidad del $30 \%$ de un incumplimiento durante el resto del plazo.

Al 1 de enero de 2016, se reconoce una previsión por deterioro del valor del $3 \%$.
- US\$ 500000 x 3\%= US\$ 15000 en función de pérdidas crediticias por 12 meses

Al 31 de diciembre de 2016, existe un aumento significativo del riesgo de incumplimiento $y$, en consecuencia, la previsión por deterioro del valor se basa en las pérdidas crediticias durante la vida útil, lo cual supone un aumento a $30 \%$.

- US\$500 $000 \times 30 \%=$ US\$ 150000

Los ingresos por intereses continúan calculándose en función de US\$500 000 .

Si las condiciones del préstamo no se hubieran cumplido al 31 de diciembre de 2016, los ingresos por intereses se habrían calculado a partir de la siguiente operación:

- US\$500000 - US\$150000=US\$350000

Las pérdidas crediticias se reconocen en resultados. Según el activo, podrán compensarse contra el importe en libros del activo en el estado de situación financiera o reconocerse como saldo crediticio por separado.

\subsection{Contabilidad de coberturas}

El nuevo modelo de coberturas sustituye al complejo y estricto enfoque actual de NIC 39 al basarse en un principio conceptual fundamental para tratar de alinear las reglas contables con las actividades de gestión del riesgo de la entidad. En otras palabras, su objetivo es transmitir la finalidad y el efecto de los instrumentos de cobertura, y las formas de utilización para gestionar el riesgo. Ello es de carácter voluntario, lo que se constituye como una excepción más a los requisitos contables normales. Aunque ciertamente las entidades no financieras no serán afectadas por las macro coberturas, lo cierto es que la opción está en vigor para cualquier tipo de empresa. 


\section{Conclusión}

- La NIIF 9 - Instrumentos Financieros establece todos los requerimientos para el reconocimiento y la medición de los activos, pasivos financieros, y algunos contratos de compra o venta de partidas no financieras de forma más sistematizada, lo cual ayuda a la mejor presentación de la información contable.

- La aplicación de la NIIF 9 en reemplazo de la NIC 39 permite a los usuarios de los estados financieros una mejor comprensión, aplicación e interpretación, al basarse en principios que la hacen menos compleja.

- Aplicar la NIIF 9 ayuda a simplificar y mejorar la contabilización de los instrumentos financieros reduciendo las asimetrías contables que se originarían debido al «Modelo de Negocio». Además, permitirá clasificar las inversiones en instrumentos financieros, las cuales en ciertos casos podrían ser sustanciales. Es decir, la falta de liquidez operativa y el mal uso del capital en ciertas pymes obliga a la utilización de la presente normativa como una opción de ayuda para activar la economía empresarial.

- Implementar la NIIF 9 en el sector industrial como nuevo modelo en cuanto al reconocimiento inicial y deterioro de los instrumentos financieros resulta trascendental, puesto que mejoraría su forma de evaluación, brindaría mayor seguridad al área Contable, y se convierte en una herramienta útil para los usuarios conceptualizando eficazmente los procesos de medición y definiendo los pasos a seguir para su aplicación en las distintas situaciones presentadas.

\section{Referencias bibliográficas}

Arias, F. G. (2012). El proyecto de investigación. Introducción a la metodología cientifica. Sexta edición. Caracas: Epísteme.

Arias Bello, M. L. (2014). NIIF 9 sobre instrumentos financieros. Comunidad contable, 29 de julio. Consulta: 15 de abril de 2018. http://www.comunidadcontable. com/BancoConocimiento/C/contrapartida_933/ contrapartida_933.asp

Cazau, P. (2012). Evolución de las relaciones entre la epistemología y la metodología de la investigación. Paradigmas, 28.

Chorafas, D. N. (2007). IFRS, valor razonable y gobierno corporativo. Bogotá: ECOE Ediciones.

Deloitte (2016). Mantente actualizado. NIIF 9 Instrumentos Financieros. Lo que hay que saber desde la perspectiva de las empresas no financieras. Grupo Técnico, IFRS Centre of Excellence, pp. 1-33.

Ferrer de la Hoz, A. M. (2013). Análisis del proceso de convergencia a Normas Internacionales de Contabilidad e Información Financiera desde los factores intrínsecos al sistema contable en Colombia. Cuadernos de Contabilidad, 14(36), 971-1007.

Higuera, J. (2014). Convergencia a las NIIF: un cambio hacia la competitividad. UAMF. Boletín de Coyuntura, 16, 1-16. Disponible en http://www. felaban.net/archivos_documentos_comites/archivo20160919185832PM.pdf

International Accounting Standards Board (2014a). NIIF 9 Instrumentos Financieros. Londres, 24 de julio.

International Accounting Standards Board (2014b). Normas internacionales de información financiera. Ministerio de Economía y Finanzas. Consulta: 17 de abril de 2018. https://www.mef.gob.pe/contenidos/ conta_publ/con_nor_co/vigentes/niif/NIIF9_2014_ v12112014.pdf 
International Accounting Standards Board. (2017). Opciones de pagos anticipados simétricos (Documento 3 de la Agenda). Londres, Reino Unido: Fundaciòn IFRS. Disponible en https:/www.ifrs.org/news-and-events/ updates/iasb-updates-spanish/january-2017/

Matiz y Asociados (2015). Modelos de negocio. Conceptos. Infoteca. Recuperado el 4 de abril de 2018 de http:// www.matizyasociados.com/conceptos-modelos-denegocio/
Superintendencia de Compañías, Valores y Seguros (2017). Indicadores económicos. Portal - Superintendencia de Compañias, Valores y Seguros. Recuperado el 4 de abril de 2018 de http://www.supercias.gob.ec/portalscvs/

Tamayo y Tamayo, M. (2012). El proceso de la investigación cientifica. México: Limusa.

Fecha de recepción: 27 de diciembre de 2017 Fecha de aceptación: 18 de abril de 2018 Correspondencia: carlos.parralesch@ug.edu.ec franklin.castillol@ug.edu.ec 